\title{
Advancing intraoperative magnetic tracing using 3D freehand magnetic particle imaging
}

\author{
Samaneh Azargoshasb ${ }^{1}$ - Lennert Molenaar ${ }^{2}$. Giuseppe Rosiello ${ }^{3,4,5}$. Tessa Buckle ${ }^{1,6}$. Danny M. van Willigen ${ }^{1}$. \\ Melissa M. van de Loosdrecht ${ }^{2} \cdot$ Mick M. Welling $^{1} \cdot$ Lejla Alic $^{2} \cdot$ Fijs W. B. van Leeuwen $^{1,4,6} \cdot$ Alexander Winter $^{7}$. \\ Matthias N. van Oosterom ${ }^{1,6}$
}

Received: 8 February 2021 / Accepted: 9 July 2021 / Published online: 31 July 2021

(c) The Author(s) 2021

\begin{abstract}
Purpose Sentinel lymph node biopsy is a routine procedure for nodal staging in penile cancer. Most commonly, this procedure is guided by radioactive tracers, providing various forms of preoperative and intraoperative guidance. This is further extended with fluorescence imaging using hybrid radioactive-fluorescence tracers. Alternatively, a magnetic-based approach has become available using superparamagnetic iron-oxide nanoparticles (SPIONs). This study investigates a novel freehand magnetic particle imaging and navigation modality (fhMPI) for intraoperative localization, along with a hybrid approach, combining magnetic and fluorescence guidance.

Materials and methods The fhMPI set-up was built with a surgical navigation device, optical tracking system and magnetometer probe. A dedicated reconstruction software based on a look-up-table method was used to reconstruct a superficial 3D volume of the SPION distribution in tissue. For fluorescence guidance, indocyanine green (ICG) was added to the SPIONs. The fhMPI modality was characterized in phantoms, ex vivo human skin and in vivo porcine surgery.

Results Phantom and human skin explants illustrated that the current fhMPI modality had a sensitivity of $2.2 \times 10^{-2} \mathrm{mg} /$ $\mathrm{mL}$ SPIONs, a resolving power of at least $7 \mathrm{~mm}$ and a depth penetration up to $1.5 \mathrm{~cm}$. Evaluation during porcine surgery showed that fhMPI allowed for an augmented reality image overlay of the tracer distribution in tissue, as well as 3D virtual navigation. Besides, using the hybrid approach, fluorescence imaging provided a visual confirmation of localized nodes.

Conclusion fhMPI is feasible in vivo, providing 3D imaging and navigation for magnetic nanoparticles in the operating room, expanding the guidance possibilities during magnetic sentinel lymph node procedures. Furthermore, the integration of ICG provides the ability to visually refine and confirm correct localization. Further clinical evaluation should verify these findings in human patients as well.
\end{abstract}

Keywords Magnetic particle-guided surgery $\cdot$ Fluorescence-guided surgery $\cdot$ Augmented reality $\cdot$ Surgical navigation · Penile cancer

Matthias N. van Oosterom

m.n.van_oosterom@lumc.nl

1 Interventional Molecular Imaging Laboratory, Department of Radiology, Leiden University Medical Center, Leiden, The Netherlands

2 Magnetic Detection \& Imaging Group, Technical Medical Centre, University of Twente, Enschede, The Netherlands

3 Department of Urology, Onze-Lieve-Vrouw Hospital, Aalst, Belgium

4 ORSI Academy, Melle, Belgium
5 Department of Urology and Division of Experimental Oncology, URI, Urological Research Institute, IRCCS San Raffaele Scientific Institute, Milan, Italy

6 Department of Urology, Netherlands Cancer Institute - Antoni van Leeuwenhoek Hospital, Amsterdam, The Netherlands

7 University Hospital for Urology, Klinikum Oldenburg, School of Medicine and Health Sciences, Carl Von Ossietzky University Oldenburg, Oldenburg, Germany 


\section{Introduction}

In cancer therapy, reliable information about the regional lymph node (LN) status is of great importance for accurate staging and optimal planning of treatment. Despite advances in non-invasive imaging [e.g. positron emission tomography (PET)], LN dissection followed by histopathological examination continues to be the most accurate modality to identify (micro-) metastases [1]. Sentinel LN (SLN) surgery is based on the premise that cancer metastases pass through one gatekeeper LN (i.e. a SLN) or a group of SLNs before spreading further [2, 3]. Focusing histopathological examination on this subset of gatekeeper LNs, it becomes feasible to perform ultrastaging of the specimens (e.g. fine serial sectioning and immunohistochemistry analysis), increasing the change to find lowvolume metastases. Sampling of these SLNs thus allows for staging of the lymphatic spread.

Advantages of such a targeted SLN biopsy include tailoring of the surgical procedure to the individual patient, allowing to distinguish between patients who need extensive nodal dissection from those who would not gain an oncological benefit from such an invasive dissection. This does not only increase the specificity of the approach, but also results in lower morbidity [4]. For these reasons, SLN biopsy has become routine for nodal staging in a.o. breast cancer, penile cancer and melanoma [5-7].

Different technologies are used for marking, preoperative visualization and intraoperative identification of SLNs. Most common and established is the use of radioactive tracers (e.g. ${ }^{99 \mathrm{~m}}$ Technetium-nanocolloid), allowing for preoperative imaging [i.e. scintigraphy and single-photon emission computed tomography/computed tomography (SPECT/CT)], as well as intraoperative detection of the SLNs using gamma detection probes and mobile gamma cameras [8]. Using the input of such gamma probes and cameras, even freehand SPECT (fhSPECT) scans have been realized in the operating room. This particular technology allows for 3D images to be generated in the operating room, a concept that further facilitates accurate navigation towards the SLNs $[9,10]$. Use of the hybrid tracer indocyanine green [ICG]- ${ }^{99 \mathrm{~m}} \mathrm{Tc}$-nanocolloid, a tracer that has proven its potential in a.o. melanoma and penile cancer [11, 12], has helped complement the abovementioned radioguidance concepts with real-time fluorescence imaging [13-15]. In parallel to these efforts, the use of magnetic tracers [e.g. superparamagnetic iron-oxide nanoparticles (SPIONs)] has also demonstrated potential in SLN procedures (e.g. penile cancer, melanoma, breast cancer and prostate cancer [16-19]. Similar to the radioguidance approach, the SLNs can be visualized preoperatively with magnetic resonance imaging (MRI) [20, 21].
Intraoperatively, the SPIONs are detected by a handheld magnetometer probe [20,21]. Similarly, as with a gamma probe in the radioguided approach, this magnetometer probe provides audible and numerical feedback with respect to the nodal tracer uptake. Unfortunately, to date, intraoperative magnetic tracing has been limited to the use of such a probe only.

To advance the intraoperative visualization of the SLNs in a magnetic approach, this study has investigated the use of a hybrid ICG-SPION approach and intraoperative freehand magnetic particle imaging (fhMPI). These technologies were evaluated in phantom measurements, ex vivo human skin explants and during SLN surgery in porcine models, focused towards applications in the groin, which is the primary lymphatic landing site for penile cancer and melanoma.

\section{Methods}

\section{Imaging tracers}

A commercially available SPION tracer, Magtrace (Endomagnetics Ltd, Cambridge, UK), was used, consisting of iron-based nanoparticles dissolved in saline. The nanoparticles contain multiple iron-oxide cores (single-core diameter: $3.5-10 \mathrm{~nm}$ ), agglomerated and encapsuled by a carboxydextran coating, bringing the hydrodynamic diameter of the particles to $45-65 \mathrm{~nm}$. To provide additional guidance during surgery and investigate a possible hybrid surgical workflow, the combined use of magnetics and fluorescence was utilized for all evaluations. To this end, $50 \mu \mathrm{L}$ of the fluorescent dye ICG $(5 \mathrm{mg} / \mathrm{mL}$ dissolved in water; Verdye, Diagnostic Green $\mathrm{GmbH}$, Aschheim-Dornach, Germany) was added to a $2 \mathrm{~mL}$ vial of SPIONs $(28 \mathrm{mg} / \mathrm{mL})$.

Additionally, to investigate possible non-covalent complex formation between ICG and SPION, the ICG-SPION mixture was investigated with a size-exclusion column, as previously described [22, 23]. To this end, $2.5 \mu \mathrm{L}$ ICG solution was added to $100 \mu \mathrm{L}$ Magtrace solution. After shaking for $30 \mathrm{~min}$ at room temperature, unbound ICG molecules were removed by purifying the mixture over a PD10 sizeexclusion column (GE-Healthcare, Chicago, USA), using saline as eluent. Both absorption and fluorescent emission spectra were recorded of the collected column fractions.

\section{Freehand magnetic particle imaging and navigation set-up}

The main components of the fhMPI imaging and navigation set-up (Fig. 1) include:

- A DeclipseSPECT navigation system (SurgicEye GmbH, Munich, Germany) with an integrated near-infrared 
Fig. 1 Schematic overview of the fhMPI imaging and navigation set-up. a Overview of the complete set-up, displaying the surgical navigation device, the handheld magnetometer probe and the near-infrared (NIR) optical tracking. b Magnification of the handheld magnetometer probe, displaying the probe reference target $\left(\mathrm{RT}^{\text {probe }}\right)$ and the patient reference target $\left(\mathrm{RT}^{\text {patient }}\right)$

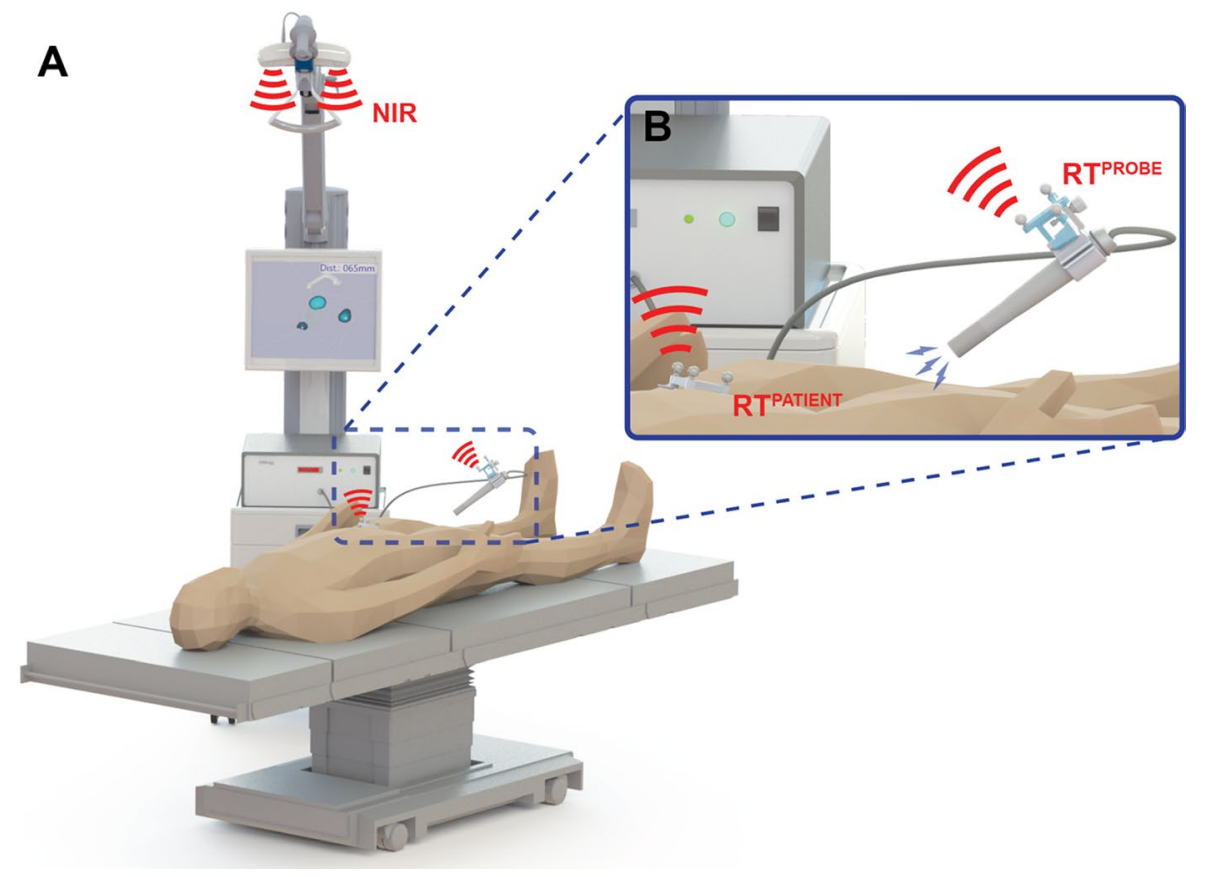

(NIR) optical tracking system (Polaris Vicra; Northern Digital Inc., Waterloo, Canada) [24];

- A $1 D$ handheld magnetometer probe (DiffMag; MD\&I group, University of Twente, The Netherlands [25]), based upon a differential magnetometry principle. The $22 \mathrm{~mm}$ diameter DiffMag probe consists of an excitation coil and a set of detection coils in a gradiometric configuration, assessing the difference between signal generated by SPIONs and background noise.

- An Arduino-based signal processing unit (Arduino Uno; Arduino AG, Italy) linking the output of the DiffMag probe to a custom version of the Declipse 6.0 software (SurgicEye $\mathrm{GmbH}$ ).

To detect the position and orientation (3D pose) of the magnetometer probe in the operating room, a three-fiducial reference target ( $\mathrm{RT}^{\text {probe }}$ ) was placed at the distal end of the probe and optically tracked (Fig. 1). This reference target consisted of a sterile part placed outside of the sterile probe cover, and a 3D printed plastic clip attached directly to the probe under the sterile cover, calibrated using previously described procedures [26]. To track the pose of the tissue of interest, a second, but geometrically different, threefiducial reference target $\left(\mathrm{RT}^{\mathrm{patient}}\right)$ was placed close to the volume of interest on a (semi-)rigid part of the scanning objective (i.e. phantom, ex vivo tissue or porcine model). During the fhMPI application, a direct line-of-sight was maintained between the NIR optical tracking system and both the RT ${ }^{\text {patient }}$ and $\mathrm{RT}^{\text {probe }}$.

Using the custom Declipse software algorithm, a superficial 3D volume was reconstructed, representing the SPION distribution in tissue (i.e. the aforementioned fhMPI scan). This reconstruction was based on a look-up-table method, common for freehand imaging as described previously [14, 27]. The look-up-table was generated by measuring the DiffMag magnetometer probe response to a SPION point source $(46 \mu \mathrm{g})$ in air over a volume of $31 \times 31 \times 9 \mathrm{~mm}$ at a resolution of $1 \mathrm{~mm}$, using a robotic $\mathrm{x}-\mathrm{y}-\mathrm{z}$ arm (Meca500, Mecademic, Montreal, Canada), similar as reported previously for freehand fluorescence imaging [14].

The settings for the eventual reconstruction were: $3 \times 3 \times 3 \mathrm{~mm}$ voxel size, $3 \mathrm{~mm}$ Gaussian filter and a $180 \times 180 \times 120 \mathrm{~mm}$ volume of interest. During fhMPI scan acquisition, the magnetometer probe was manually moved over the tissue in the volume of interest, measuring at as many different positions and orientations as possible, keeping the total acquisition time below $5 \mathrm{~min}$. Even though the current magnetometer probe employs differential magnetometry to correct for interference by nearby metal objects (e.g. surgical clamps) [25], some 'noise counts' are still observed when the probe is moved dynamically. To minimize noise in the fhMPI scans during acquisition, magnetometer measurements below 20 counts were excluded.

\section{Characterization of the freehand magnetic particle imaging and navigation modality}

Feasibility of the fluorescent and magnetic particle tracking in human tissue was investigated using explanted human skin samples. To this end, 3 deposits of each $100 \mu \mathrm{L}$ ICGSPION tracer were injected subcutaneously. In this setting, each deposit roughly represented a typical SLN uptake value 
as found in prostate/penile cancer (i.e. $5 \%$ of the typically injected dose [23, 28]). FhMPI imaging and navigation were evaluated, as well as fluorescence imaging using a PDE-mod open surgery fluorescence camera (Hamamatsu Photonics k.k., Hamamatsu, Japan).

To investigate the sensitivity of the novel fhMPI modality, a well plate was used to create a dilution series of SPION tracer. Divided over 14 wells, each well was filled with $100 \mu \mathrm{L}$ of ICG-SPION, ranging from $2.8 \mathrm{mg} / \mathrm{mL}$ down to $1.7 \times 10^{-4} \mathrm{mg} / \mathrm{mL}$ using a human serum albumin solution ( $40 \mathrm{mg} / \mathrm{mL}$ in $\mathrm{H}_{2} \mathrm{O}$ ). For each well, a background-corrected magnetometer signal was recorded and a fhMPI scan was made, evaluating the minimal tracer concentration for successful reconstruction. All measurements were taken in triplicate.

The resolving power of the fhMPI modality was assessed using a plastic plate phantom [14], containing 7 well pairs with each a decreasing distance between the edges (ranging from 18 to $4 \mathrm{~mm}$ ). Each of the individual wells was filled with $100 \mu \mathrm{L}$ ICG-SPION tracer. With the unobstructed liquid level present at the surface of the phantom (i.e. a depth of $0 \mathrm{~cm}$ ), any depth penetration effects were avoided in this set-up. The fhMPI scan was acquired in triplicate for each well pair.

Depth penetration of fhMPI was evaluated using explanted human skin. $100 \mu \mathrm{L}$ of ICG-SPION was inserted at different depths into the tissue (i.e. $0,0.5,1,1.5$ and $2 \mathrm{~cm}$ ) and investigated using magnetometer counts and the novel fhMPI method.

\section{In vivo porcine evaluation}

In vivo feasibility of the fhMPI set-up was investigated in porcine models $(n=2$, four groins; weight per animal approximately $40 \mathrm{~kg}$ ). ICG-SPION was administered in the operating room, using subcutaneous injections in both legs (0.5 mL each). Immediately after administration, the injection sites were massaged for $\sim 15$ min to promote lymphatic drainage. The time between injection and localization was $45 \mathrm{~min}$. The $\mathrm{RT}^{\text {patient }}$ was placed on the skin surface at the pubic bone location. The localization started with transcutaneous magnetic tracing using the magnetometer probe, followed by fhMPI scan acquisition of the anatomy of interest and an additional check with fluorescence imaging (Firefly fluorescence endoscope and endoscope plus models, DaVinci Xi, Intuitive Inc., Sunnyvale CA, USA). The information of these modalities was used to guide the incision and localize individual SLNs. Upon exposure of the node, fluorescence imaging was performed again to confirm the nodal target. After surgical retrieval, the SLN specimens were also imaged ex vivo. Animal experiments were performed under approval of the local ethical committee.

\section{Pathology}

To confirm the presence of SPIONs in the excised SLNs, the surgical specimens were fixed in $4 \%$ buffered formaldehyde for 1 week at room temperature and then processed for paraffin embedding. Next, sections of $4 \mu \mathrm{m}$ were cut and dried overnight at $37{ }^{\circ} \mathrm{C}$. Perl's Prussian blue iron staining was used to investigate for SPION presence, while the nuclei were stained with nuclear fast red to provide anatomical context.

\section{Results}

\section{Hybrid SPION tracer}

Similar as was shown for ICG- ${ }^{99 \mathrm{~m}} \mathrm{Tc}$-nanocolloid $[22$, 23], after purifying ICG-SPION over a size-exclusion column, ICG fluorescence should be minimally present in the first eluting column fraction, unless ICG molecules were directly bound to SPION particles. Figure 2 visualizes the fluorescence intensity of the first eluting fraction for an ICG column and an ICG-SPION column, clearly showing an increase in fluorescence measured at $810 \mathrm{~nm}$. Analysing this fraction, we were able to identify the distinct fluorescence absorption and emission signatures of ICG in the fraction that contains SPIONs. This indicates that at least part of the ICG has bound non-covalently to the SPION nanoparticles,

\section{Fluorescence intensity first fraction post-column}

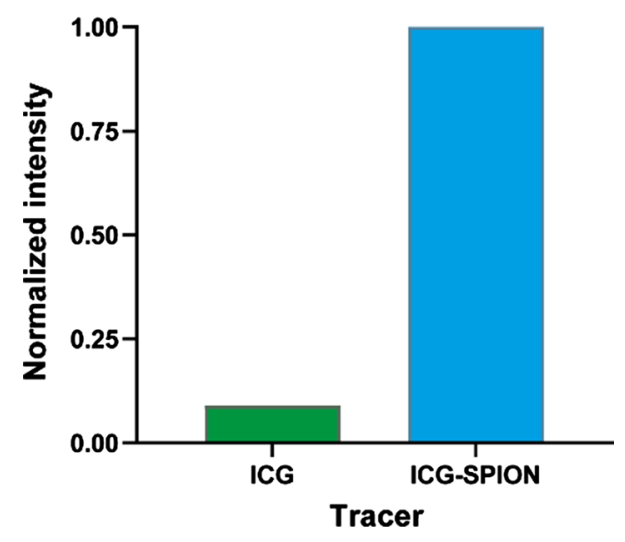

Fig. 2 Visualization of the size-exclusion column measurements for ICG-SPION. Fluorescence intensity is shown of the first fraction after purification of a column with ICG and a column with ICG-SPION. This clearly shows a rise of fluorescence emission at $810 \mathrm{~nm}$ for ICGSPION, indicating that at least part of the ICG has bound non-covalently to the SPION particles. The intensity values were corrected by subtracting the SPION background intensity and normalized to that of ICG-SPION 


\section{Characterization of the freehand magnetic particle imaging and navigation modality}

Figure 3 visualizes the first feasibility of fhMPI imaging and navigation in human skin tissue. Scan reconstruction was performed 'on-the-fly', immediately visualizing and updating the results during scan acquisition. The reconstructed fhMPI scans were visualized in two ways: (1) an augmented reality overlay on the target anatomy, providing the surgeon with insight as to where the SPION-hotspots were found in the tissue (Fig. 3a); (2) a 3D virtual reality view, which could be used to navigate the magnetometer probe towards the individual SPION-hotspots (Fig. 3b), while the real-time read-out of the probe provided audible feedback. Finally, fluorescence imaging confirmed the location of the SPION-hotspots (Fig. 3c).

Further evaluation of the fhMPI imaging characteristics is visualized in Fig. 4. The success of fhMPI imaging was strongly related to the sensitivity of the magnetometer probe used, indicating a cut-off at $2.2 \times 10^{-2} \mathrm{mg} /$ $\mathrm{mL}$ (Fig. 4a). At this range, the magnetometer probe counts dropped below 20. Measurements for the resolving power revealed that two lymph-node-like targets could be resolved as individual targets with an edge-to-edge distance $\geq 7 \mathrm{~mm} \quad$ (Fig. 4c). The fhMPI depth penetration allowed to find tracer hotspots up to roughly $1.5 \mathrm{~cm}$ deep (Fig. 4b).
Fig. 3 Ex vivo human skin evaluation of the fhMPI modality. a Augmented reality overlay of the fhMPI scan on the target anatomy. The calculated distance between the magnetometer probe tip and the centre of the tracer hotspot that is pointed at, is displayed in the upper right corner of the image. b Virtual reality navigation towards the tracer hotspots detected in the image, again with the distance towards the hotspots displayed in the upper right corner of the image. c Confirmation of the surgical target using fluorescence imaging
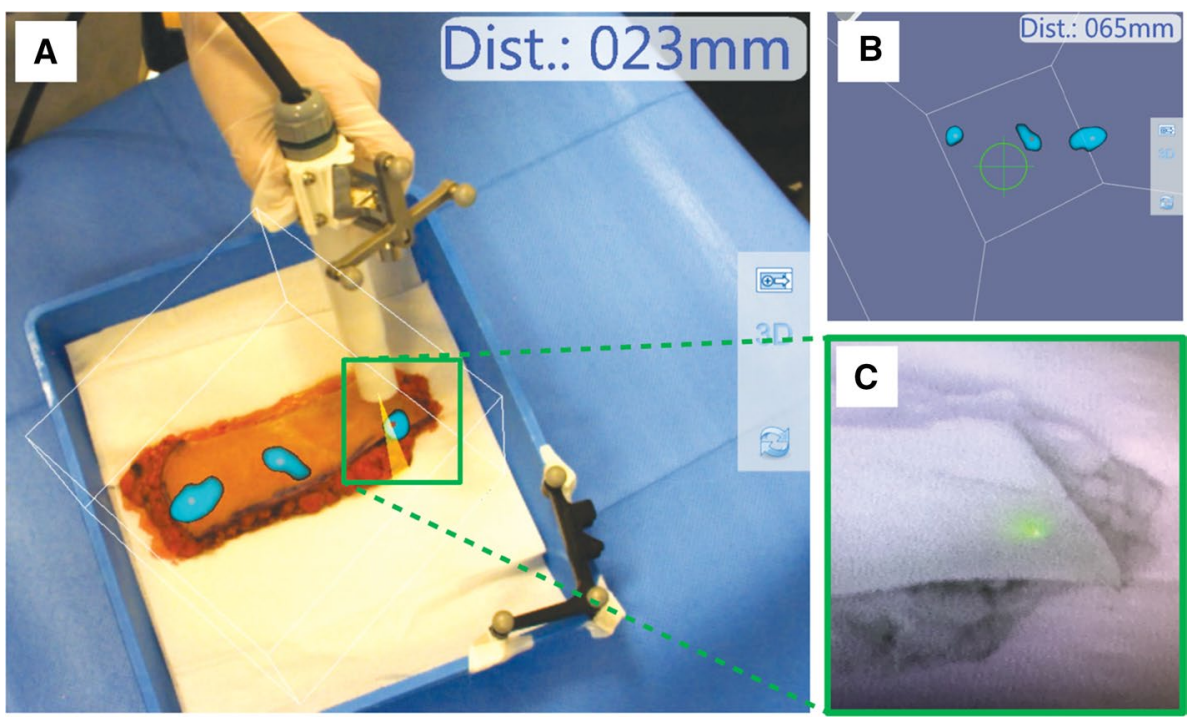

A

Fig. 4 Characterization of the fhMPI modality. a fhMPI sensitivity, displaying the minimal SPION concentration needed for successful imaging. b fhMPI depth penetration, showing successful scanning is still possible up to $1.5 \mathrm{~cm}$. c Overview of the resolving power results, showing that two lymph-nodelike targets were still resolvable when the edges of the nodes $7 \mathrm{~mm}$ were at a distance of at least

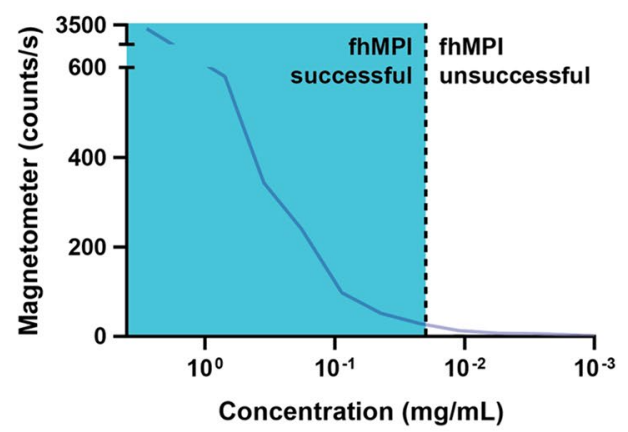

B

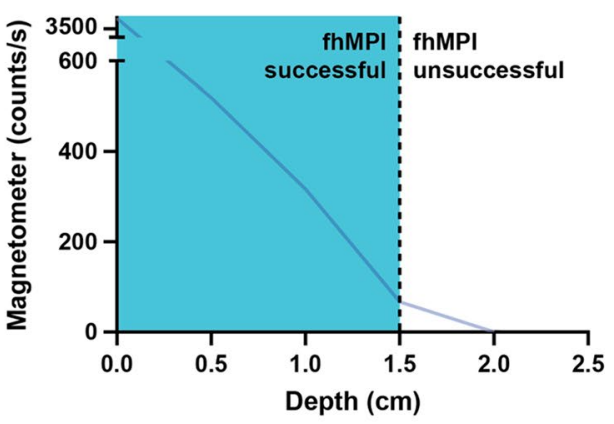

C fhMPI resolving power

\begin{tabular}{|l|l|l|l|l|l|l|}
\hline $18 \mathrm{~mm}$ & $13 \mathrm{~mm}$ & $8 \mathrm{~mm}$ & $7 \mathrm{~mm}$ & $6 \mathrm{~mm}$ & $5 \mathrm{~mm}$ & $4 \mathrm{~mm}$ \\
\hline & 0 & 0 & 0 & & & \\
0 & 0 & 0 & 0 & & \\
\hline
\end{tabular}




\section{In vivo porcine evaluation and pathology}

Figure 5 shows an overview of the in vivo fhMPI evaluation in the porcine models. In the four groins, a total of five SLNs were localized in the superficial inguinal lymph node groups. There was non-visualization in one groin, something that is in line with clinical SLN procedures [11].

Localization of the SLNs started with probing the unopened groins of the pigs using the magnetometer probe, relying on the probe's audible feedback. Local signals became stronger when the probe was pressed into the tissue. Once a potential hotspot was identified, a fhMPI scan was acquired $(\sim 3 \mathrm{~min})$. The ability to visualize a clear hotspot in augmented reality using fhMPI helped boost the surgical confidence with regard to the nodal location (Fig. 5c). Virtual reality navigation based on this fhMPI scan allowed for an estimation of the SLN depths prior to incision, as well as guidance during the incision (Fig. 5d). All the SLNs localized using magnetic tracing were located too deep below the skin surface to allow for direct identification using fluorescence imaging. This changed once the node locations were exposed, which allowed for confirmation with fluorescence imaging (Fig. 5e). Ex vivo examinations revealed that all resected nodes were magnetic and fluorescent. They also all had a (slightly) brownish colouration, which is typical for SPION uptake in the lymph nodes [29]. Histopathologic
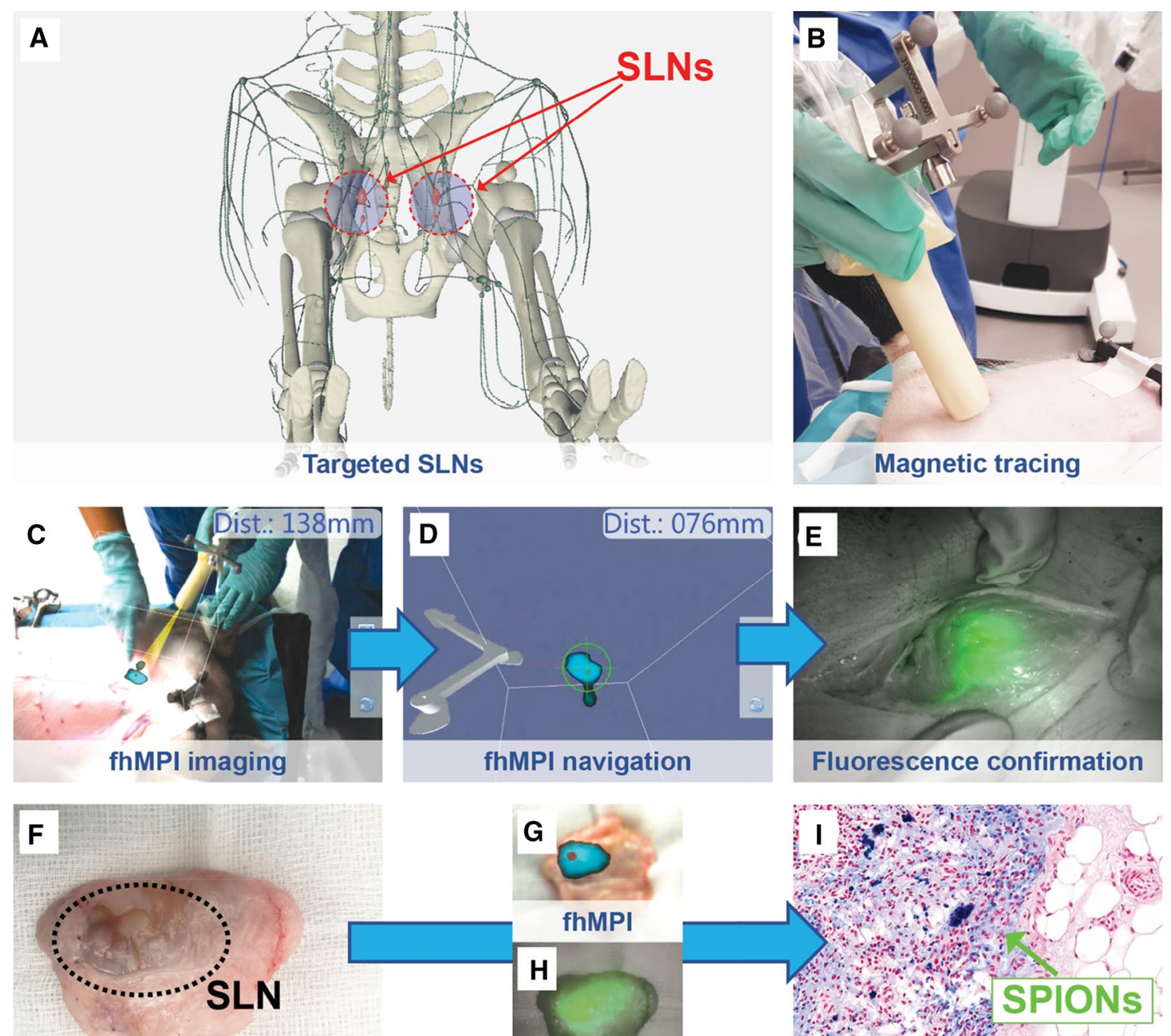

Excised SLN specimen
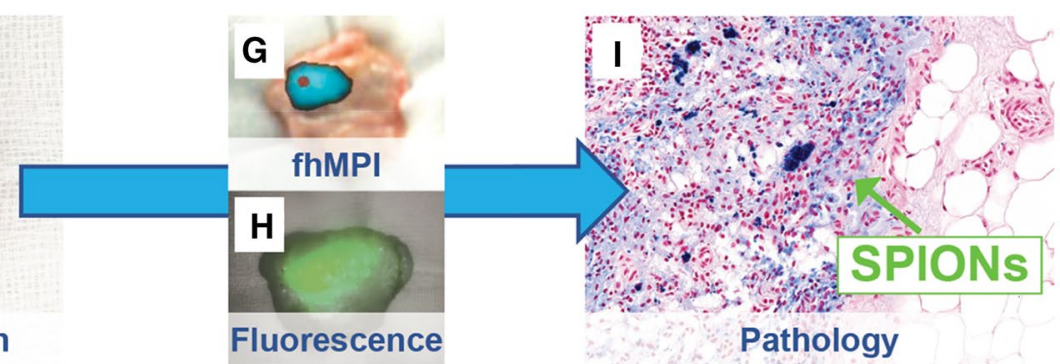

Fig. 5 In vivo porcine evaluation of the fhMPI modality. a Targeted SLNs displayed within the anatomy. b Surgical localization starts with rough magnetic tracing. $\mathbf{c}$ This is followed with a fhMPI scan in the area of interest, displaying the SLN location registered as an augmented reality overlay on the anatomy. d Virtual reality navigation towards the lymph node location. e Fluorescence imaging con-

firms the lymph node location once the tissue is exposed. $\mathbf{f}$ Ex vivo evaluation depicts a typical SPION-brownish colour in the specimen. g SPION uptake confirmed with fhMPI. h SPION uptake confirmed with fluorescence. i Histopathology confirms the SPIONs (in blue) are indeed in the resected lymph node 
examination of the specimens additionally confirmed the presence of SPIONs within the lymphatic tissue (Fig. 5i).

\section{Discussion}

In this study, we've successfully built and evaluated a novel imaging and navigation modality (i.e. fhMPI) for the intraoperative detection of SPION tracers. This could expand the guidance possibilities during magnetic SLN procedures for various oncological indications (e.g. penile, breast, melanoma, head-and-neck, prostate and vulva cancer). By creating the possibility of hybrid imaging, fhMPI and the related navigation options, intraoperative magnetic imaging is now moving towards the technical advances previously made in radioguided SLN resections.

With the introduction of fhMPI, the family of freehand molecular imaging has been expanded with a fourth modality. As previously reported for fhSPECT [9, 24], freehand beta particle imaging (fhBeta) [30-32] and freehand fluorescence (fhFluo) [14], there are some interesting characteristics that place fhMPI in the context of the other freehand methods. First, image resolving power is highest with fhFluo with respect to fhSPECT and fhMPI $(1 \mathrm{~mm}, 6 \mathrm{~mm}$ and $7 \mathrm{~mm}$, respectively) [14]. The image resolving power for fhBeta is not reported, but expected to be around $1 \mathrm{~mm}$ [8]. Second, depth penetration of fhMPI and fhSPECT is higher than with fhFluo $(1.5 \mathrm{~cm},>1.5 \mathrm{~cm}$ and $0.5 \mathrm{~cm}$, respectively) [14]. The depth penetration of fhBeta is not reported, but expected to be only a few mm's [8]. Based on these parameters, fhMPI makes an interesting clinical addition to the available options.

All SLNs found in the in vivo porcine models were both magnetic and fluorescent. Hence, this feature opens up the possibility to see if a hybrid concept involving MRI, magnetic tracing and fluorescence imaging could provide an alternative to those using fluorescence imaging and radioguided surgery [15].

Limiting in this study was the lack of porcine preoperative imaging, making it possible that not all stained SLNs were localized during surgery. When the concept is evaluated in clinical routine, however, preoperative SLN mapping can be realized using MRI, providing a roadmap for surgery, something that has proven critical in the radioactive SLN approach [13].

\section{Conclusions}

Freehand magnetic particle imaging is feasible in vivo, providing a $3 \mathrm{D}$ imaging and navigation modality for magnetic nanoparticles in the operating room. In addition, our findings show that the additional use of ICG provides the ability to refine the procedure via integrated fluorescence imaging. Further clinical evaluation should confirm this also provides added benefit in human patients.

Acknowledgements We acknowledge the ORSI Academy staff (Belgium) for assistance during surgical evaluations.

Funding This study was funded by an NWO-TTW-VICI Grant (\#16141).

Data availability Data are available on reasonable request.

Code availability Not applicable.

\section{Declarations}

Conflict of interest During this research, GR and FvL were (partially) affiliated with ORSI Academy. The authors declare that they have no further conflicts of interest.

Consent to participate Not applicable.

Consent for publication Not applicable.

Ethics approval Experiments in pigs were approved by the ethical board of the University of Ghent prior to execution (\#EC2019/79). Pigs were housed at the animal facility at ORSI Academy (Melle, Belgium). Experiments were performed in accordance with the Experiments on Animals Act (Wod, 2014), the applicable legislation in Belgium and in accordance with the European guidelines (EU directive no. 2010/63/ $\mathrm{EU})$ regarding the protection of animals used for scientific purposes.

Open Access This article is licensed under a Creative Commons Attribution 4.0 International License, which permits use, sharing, adaptation, distribution and reproduction in any medium or format, as long as you give appropriate credit to the original author(s) and the source, provide a link to the Creative Commons licence, and indicate if changes were made. The images or other third party material in this article are included in the article's Creative Commons licence, unless indicated otherwise in a credit line to the material. If material is not included in the article's Creative Commons licence and your intended use is not permitted by statutory regulation or exceeds the permitted use, you will need to obtain permission directly from the copyright holder. To view a copy of this licence, visit http://creativecommons.org/licenses/by/4.0/.

\section{References}

1. van Leeuwen FWB, Winter A, van Der Poel HG, Eiber M, Suardi N, Graefen M, Wawroschek F, Maurer T (2019) Technologies for image-guided surgery for managing lymphatic metastases in prostate cancer. Nat Rev Urol 16(3):159-171

2. Zeidman I, Buss JM (1954) Experimental studies on the spread of cancer in the lymphatic system: I. Effectiveness of the lymph node as a barrier to the passage of embolic tumor cells. Cancer Res 14(5):403-405

3. Gould EA, Winship T, Philbin PH, Kerr HH (1960) Observations on a "sentinel node" in cancer of the parotid. Cancer 13(1):77-78

4. Wawroschek F, Wagner T, Hamm M, Weckermann D, Vogt H, Märkl B, Gordijn R, Harzmann R (2003) The influence of serial sections, immunohistochemistry, and extension of pelvic lymph 
node dissection on the lymph node status in clinically localized prostate cancer. Eur Urol 43(2):132-137

5. Hakenberg O, Compérat E, Minhas S, Necchi A, Protzel C, Watkin N (2020) EAU guidelines on penile cancer. In: EAU guidelines. EAU Annual Congress Amsterdam

6. Miltenburg DM, Miller C, Karamlou TB, Brunicardi FC (1999) Meta-analysis of sentinel lymph node biopsy in breast cancer. J Surg Res 84(2):138-142

7. Morton DL, Thompson JF, Cochran AJ, Mozzillo N, Nieweg OE, Roses DF, Hoekstra HJ, Karakousis CP, Puleo CA, Coventry BJ (2014) Final trial report of sentinel-node biopsy versus nodal observation in melanoma. N Engl J Med 370(7):599-609

8. Van Oosterom MN, Rietbergen DD, Welling MM, Van Der Poel HG, Maurer T, van Leeuwen FWB (2019) Recent advances in nuclear and hybrid detection modalities for image-guided surgery. Expert Rev Med Devices 16(8):711-734

9. Bluemel C, Matthies P, Herrmann K, Povoski SP (2016) 3D scintigraphic imaging and navigation in radioguided surgery: freehand SPECT technology and its clinical applications. Expert Rev Med Devices 13(4):339-351

10. van Oosterom MN, van der Poel HG, Navab N, van de Velde CJ, van Leeuwen FWB (2018) Computer-assisted surgery: virtualand augmented-reality displays for navigation during urological interventions. Curr Opin Urol 28(2):205-213

11. Dell'Oglio P, de Vries HM, Mazzone E, KleinJan GH, Donswijk ML, van der Poel HG, Horenblas S, van Leeuwen FWB, Brouwer OR (2020) Hybrid Indocyanine Green-99mTc-nanocolloid for single-photon emission computed tomography and combined radio-and fluorescence-guided sentinel node biopsy in penile cancer: results of 740 inguinal basins assessed at a single institution. Eur. Urol.

12. van den Berg NS, Brouwer OR, Schaafsma BE, Mathéron HM, Klop WMC, Balm AJ, van Tinteren H, Nieweg OE, van Leeuwen FWB, Valdés Olmos RA (2015) Multimodal surgical guidance during sentinel node biopsy for Melanoma: combined gamma tracing and fluorescence imaging of the sentinel node through use of the hybrid tracer indocyanine green-99mTc-nanocolloid. Radiology 275(2):521-529

13. Meershoek P, Buckle T, van Oosterom MN, KleinJan GH, van der Poel HG, van Leeuwen FWB (2020) Can intraoperative fluorescence imaging identify all lesions while the road map created by preoperative nuclear imaging is masked? J Nucl Med 61(6):834-841

14. van Oosterom MN, Meershoek P, Welling MM, Pinto F, Matthies P, Simon H, Wendler T, Navab N, van de Velde CJ, van der Poel HG (2019) Extending the hybrid surgical guidance concept with freehand fluorescence tomography. IEEE Trans Med Imaging 39(1):226-235

15. KleinJan GH, Van Werkhoven E, van Den Berg N, Karakullukcu M, Zijlmans H, Van Der Hage J, Van De Wiel B, Buckle T, Klop W, Horenblas S (2018) The best of both worlds: a hybrid approach for optimal pre-and intraoperative identification of sentinel lymph nodes. Eur J Nucl Med Mol Imaging 45(11):1915-1925

16. Douek M, Klaase J, Monypenny I, Kothari A, Zechmeister K, Brown D, Wyld L, Drew P, Garmo H, Agbaje O (2014) Sentinel node biopsy using a magnetic tracer versus standard technique: the SentiMAG Multicentre Trial. Ann Surg Oncol 21(4):1237-1245

17. Winter A, Woenkhaus J, Wawroschek F (2014) A novel method for intraoperative sentinel lymph node detection in prostate cancer patients using superparamagnetic iron oxide nanoparticles and a handheld magnetometer: the initial clinical experience. Ann Surg Oncol 21(13):4390-4396

18. Winter A, Kowald T, Engels S, Wawroschek F (2020) Magnetic resonance sentinel lymph node imaging and magnetometer-guided intraoperative detection in penile cancer, using superparamagnetic iron oxide nanoparticles: first results. Urol Int 104(3-4):177-180
19. Piñero-Madrona A, Nicolás-Ruiz F, Rull-Ortuño R, Vidal-Sicart S, Cabañas-Montero J, Rioja-Martín ME, Rodríguez-Fernández R, GilOlarte MÁ, González-García B, Sánchez JHG (2020) Correlation between ferromagnetic and isotopic tracers for sentinel lymph node detection in cutaneous melanoma: IMINEM study. J Surg Oncol

20. Winter A, Chavan A, Wawroschek F (2018) Magnetic resonance imaging of sentinel lymph nodes using intraprostatic injection of superparamagnetic iron oxide nanoparticles in prostate cancer patients: first-in-human results. Eur Urol 73:813-814

21. Pouw JJ, Grootendorst MR, Bezooijen R, Klazen CA, De Bruin WI, Klaase JM, Hall-Craggs MA, Douek M, ten Haken B (2015) Pre-operative sentinel lymph node localization in breast cancer with superparamagnetic iron oxide MRI: the SentiMAG Multicentre Trial imaging subprotocol. Br J Radiol 88(1056):20150634

22. Bunschoten A, Buckle T, Kuil J, Luker GD, Luker KE, Nieweg OE, van Leeuwen FWB (2012) Targeted non-covalent self-assembled nanoparticles based on human serum albumin. Biomaterials 33(3):867-875

23. KleinJan GH, Bunschoten A, van den Berg NS, Olmos RAV, Klop WMC, Horenblas S, van der Poel HG, Wester H-J, van Leeuwen FWB (2016) Fluorescence guided surgery and tracer-dose, fact or fiction? Eur J Nucl Med Mol Imaging 43(10):1857-1867

24. Wendler T, Herrmann K, Schnelzer A, Lasser T, Traub J, Kutter O, Ehlerding A, Scheidhauer K, Schuster T, Kiechle M (2010) First demonstration of 3-D lymphatic mapping in breast cancer using freehand SPECT. Eur J Nucl Med Mol Imaging 37(8):1452-1461

25. Waanders S, Visscher M, Wildeboer R, Oderkerk T, Krooshoop HJ, ten Haken B (2016) A handheld SPIO-based sentinel lymph node mapping device using differential magnetometry. Phys Med Biol 61(22):8120

26. van Oosterom MN, Engelen MA, van den Berg NS, KleinJan GH, van der Poel HG, Wendler T, van de Velde CJH, Navab N, van Leeuwen FWB (2016) Navigation of a robot-integrated fluorescence laparoscope in preoperative SPECT/CT and intraoperative freehand SPECT imaging data: a phantom study. J Biomed Opt 21(8):086008

27. Hartl A, Shakir DI, Lasser T, Ziegler SI, Navab N (2015) Detection models for freehand spect reconstruction. Phys Med Biol 60(3): 1031

28. Wawroschek F, Wengenmair H, Senekowitsch-Schmidtke R, Hamm M, Henke J, Schönberger T, Hauser A, Erhardt W, Harzmann R (2003) Prostate lymphoscintigraphy for sentinel lymph node identification in canines: reproducibility, uptake, and biokinetics depending on different injection strategies. Urol Res 31(3): 152-158

29. Cousins A, Balalis G, Thompson S, Morales DF, Mohtar A, Wedding A, Thierry B (2015) Novel handheld magnetometer probe based on magnetic tunnelling junction sensors for intraoperative sentinel lymph node identification. Sci Rep 5:10842

30. Wendler T, Hartl A, Lasser T, Traub J, Daghighian F, Ziegler SI, Navab N (2007) Towards intra-operative 3D nuclear imaging: reconstruction of $3 \mathrm{D}$ radioactive distributions using tracked gamma probes. In: International conference on medical image computing and computer-assisted intervention. Springer, pp 909-917

31. Wendler T, Traub J, Ziegler SI, Navab N (2006) Navigated three dimensional beta probe for optimal cancer resection. In: International conference on medical image computing and computerassisted intervention, Springer, pp 561-569

32. Monge F, Shakir DI, Le Jeune F, Morandi X, Navab N, Jannin $P$ (2017) Acquisition models in intraoperative positron surface imaging. Int J Comput Assist Radiol Surg 12(4):691-703

Publisher's Note Springer Nature remains neutral with regard to jurisdictional claims in published maps and institutional affiliations. 